

В. А. Рубаков, С. М. Сибиряков, Распад ложного вакуума в пространствевремени де Ситтера, ТМФ, 1999, том 120, номер 3, 451-472

DOI: https://doi.org/10.4213/tmf790

Использование Общероссийского математического портала Math-Net.Ru подразумевает, что вы прочитали и согласны с пользовательским соглашением

http://www.mathnet.ru/rus/agreement

Параметры загрузки:

IP: 54.237 .206 .68

26 апреля 2023 г., 18:10:19

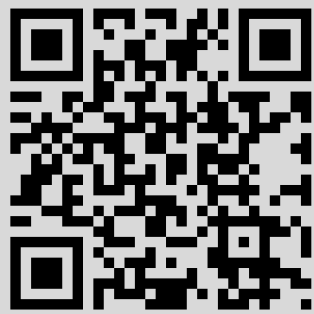




\author{
ТЕОРЕТИЧЕСКАЯ \\ И МАТЕМАТИЧЕСКАЯ \\ ФИЗИКА \\ Том 120, № 3 \\ сентябрь, 1999
}

(C) 1999 г.

В. А. Рубаков* , С.М. Сибиряков*

\title{
РАСПАД ЛОЖНОГО ВАКУУМА \\ В ПРОСТРАНСТВЕ-ВРЕМЕНИ \\ ДЕ СИТТЕРА
}

На примере распада метастабильного состояния скалярного поля (конформный вакуум скалярных частиц над ложным классическим вакуумом) во внешней метрике де Ситтера предложен метод учета начального состояния квантового поля при квазиклассическом вычислении функционального интеграла в искривленном пространстве-времени. С помощью этого метода получено обоснование подхода Коулмена-де Люччиа к вычислению вероятности распада. Дана интерпретация инстантона Хокинга-Мосса как предела инстантонов со связью. Получено, что в пространстве де Ситтера может происходить обратный процесс перехода из истинного вакуума в ложный, и найдено выражение для соответствующей вероятности.

\section{1. ВВЕДЕНИЕ}

В связи с инфляционными космологическими теориями, а также теориями, объясняющими генерацию наблюдаемой барионной асимметрии при помоши фазовых переходов первого рода в ранней Вселенной, большой интерес представляет проблема описания распада ложного вакуума в рамках квантовой теории поля. Довольно давно было установлено, что в моделях со слабой связью процесс туннелирования поля из ложного вакуума в истинный является квазиклассическим, и был развит надежный метод вычисления вероятности этого процесса для случая скалярного поля в пространстве Минковского [1-3]. Было показано, что распад ложного вакуума происходит посредством образования пузырей стабильной фазы (соответствующей истинному вакууму) среди метастабильной (ложный вакуум) и что задача вычисления показателя экспоненты в квазиклассическом выражении для вероятности образования пузыря в единицу времени в единице объема:

$$
\Gamma=A e^{-B},
$$

сводится к решению классических уравнений поля в евклидовом пространстве-времени с граничными условиями обрашения поля в ложный вакуум при стремлении евклидова времени $\tau$ к $\pm \infty$. При этом $B$ равно евклидову действию, вычисленному на нетривиальном (не равном тождественно ложному вакууму) решении указанной задачи, за вычетом евклидова действия на тривиальном решении. Если граничная задача имеет несколько

\footnotetext{
* Институт ядерных исследований РАН, Москва, Россия
} 
нетривиальных решений, то для вычисления $B$ должно быть выбрано решение, действие для которого минимально. Было показано, что при любом потенциале скалярного поля сушествует $O(4)$-симметричное решение ( “евклидов пузырь" ) [3] и что оно минимизирует евклидово действие в классе нетривиальных решений [4]. Аналитическоепродолжение этого решения в область чисто мнимого $\tau=i t$ дает конфигурацию пузыря после его образования как функцию физического времени $t$. Перечисленные результаты были получены в рамках метода функционального интегрирования. Стоит отметить, что, хотя в настоящей статье мы занимаемся вопросом вычисления только главной квазиклассической экспоненты, этот метод позволяет найти также и предэкспоненциальный множитель $A[3]$.

Задача учета гравитационных эффектов при образовании и дальнейшем расширении пузырей была получена Коулменом и де Люччиа [5]. Ими было сделано предположение, что по аналогии со случаем плоского пространства-времени вероятность образования пузыря определяется $O(4)$-симметричным решением системы уравнений, состоящей из уравнения Клейна-Гордона-Фока и уравнений Эйнштейна для скалярного поля и римановой метрики с евклидовой сигнатурой. Показатель экспоненты $B$ равен по-прежнему разности евклидовых действий на нетривиальном и тривиальном решениях этой системы (тривиальное решение - это решение, в котором скалярное поле тождественно равно своему значению в ложном вакууме), где действие теперь включает два члена: действие скалярного поля в искривленном пространстве и действие гравитационного поля. При этом можно показать [6], что для неотрицательного скалярного потенциала (этот случай наиболее интересен с точки зрения инфляционных теорий) метрика данного решения описывает пространство с топологией четырехмерной сферы $S^{4}$. Аналитическое продолжение риманова решения в пространство с сигнатурой метрики Минковского дает эволюцию пузыря в реальном пространстве-времени, причем в удаленном прошлом пространство-время характеризуется метрикой де Ситтера.

Нам представляется, что этот привлекательный и простой подход нуждается в более подробном обосновании. Дело прежде всего в том, что он целиком основан на аналогии с теорией поля в плоском пространстве-времени. Эта аналогия, вообше говоря, могла бы не быть справедливой для сильно искривленного пространственно-временного многообразия. В частности, для целого класса скалярных потенциалов единственным $O(4)$-симметричным евклидовым решением является инстантон Хокинга-Мосса [7], представляющий собой четырехмерную сферу, на которой значение скалярного поля постоянно. Такой инстантон кардинально отличается от "евклидова пузыря" в плоском пространстве-времени, а его непосредственное аналитическое продолжение в пространство-время с сигнатурой Минковского приводит к однородному скалярному полю, а не к пузырю истинного вакуума в ложном.

Кроме того, само понятие ложного вакуума в искривленном пространстве-времени требует уточнения. Помимо задания классического вакуума - среднего значения скалярного поля - необходимо зафиксировать состояние, в котором находятся квантовые возбуждения этого поля. Действительно, даже в теории свободных полей в искривленном пространстве-времени вакуумное состояние определено неоднозначно и зависит от выбора полевых мод, по которым производится разложение полевых операторов (см., например, $[8,9])$. Поэтому представляет интерес вопрос, какому именно выбору кван- 
тового вакуума соответствуют рецепты Коулмена-де Люччиа и Хокинга-Мосса ${ }^{1)}$.

При определенных соотношениях между параметрами теории адекватным для изучения части этих проблем является стохастический подход [11]. В рамках этого подхода было продемонстрировано $[11,12]$, что действие инстантонов Хокинга-Мосса совпадает с показателем экспоненты распада ложного классического вакуума, когда состояние квантовых возбуждений над ним - это конформный вакуум скалярного поля. В то же время роль самого инстантонного решения при стохастическом подходе остается неясной.

Естественным шагом в решении отмеченных выше проблем является изучение распада ложного вакуума в предельном случае, когда характерный масштаб изменения скалярного потенциала в процессе туннелирования мал по сравнению с самим потенциалом. В этом случае изменением метрики при образовании пузыря можно пренебречь, и мы приходим к квантовой теории поля во внешнем искривленном пространстве, а конкретно в пространстве де Ситтера. Несомненное достоинство данного приближения состоит в том, что не возникает необходимости учитывать эффекты квантовой гравитации, т.е. задача может быть поставлена и решена целиком в рамках обычной квантовой теории поля, хотя и в искривленном пространстве-времени. Мы ограничимся выбором квантового состояния над ложным классическим вакуумом в виде конформного вакуума скалярных частиц. Такое состояние естественно возникает в инфляционных теориях. При этих условиях мы покажем, что распад метастабильного состояния действительно описывается инстантоном Коулмена-де Люччиа (в моделях, где он сушествует), дадим интерпретацию инстантона Хокинга-Мосса как предела инстантонов со связью и обсудим решения, не имеющие аналогов в плоском пространстве-времени и соответствующие туннелированию из истинного вакуума в ложный.

\section{2. ПОСТАНОВКА ЗАДАЧИ}

Рассмотрим теорию скалярного поля $\phi$ с потенциалом, изображенным на рис. 1. Состояние $\phi=0$ соответствует ложному, а $\phi=\phi_{+}-$истинному вакууму; эти вакуумы разделены локальным максимумом скалярного потенциала $V\left(\phi_{0}\right)$. Обсудим сначала следуюший вопрос: имеет ли вообше смысл рассматривать влияние гравитации на туннелирование в приближении внешней метрики? Введем массовый масштаб $M$, характеризуюший изменение потенциала на интервале между двумя вакуумами. Гравитационное поле характеризуется постоянной Хаббла $H$. Гравитационные эффекты существенны для описания образования пузыря, если отношение $H / M$ порядка или больше единицы, а приближение внешней метрики де Ситтера справедливо при

$$
\frac{M^{4}}{V\left(\phi_{+}\right)} \ll 1
$$

\footnotetext{
1) Не исключено, что различные выборы состояния квантовых возбуждений над ложным классическим вакуумом могут приводить к существенно отличающимся вероятностям его распада. На это указывает следующее соображение. Известно (см., например, [9]), что уже в теории свободных полей в искривленном пространстве-времени нередко возникает представление от эффективной температуре, характеризующей тот или иной квантовый вакуум. Кроме того, показатель экспоненты распада метастабильного состояния в пространстве Минковского при конечных температурах имеет существенную температурную зависимость [10].
} 




Рис. 1

Из уравнения Эйнштейна

$$
H^{2}=\frac{8 \pi}{3 M_{\mathrm{P} 1}^{2}} V\left(\phi_{+}\right),
$$

где $M_{\mathrm{Pl}}$ - масса Планка, имеем

$$
\frac{H}{M}=\frac{1}{M M_{\mathrm{Pl}}} \sqrt{\frac{8 \pi V\left(\phi_{+}\right)}{3}},
$$

что вполне может быть порядка единицы, если

$$
\frac{V\left(\phi_{+}\right)^{\frac{1}{4}}}{M_{\mathrm{Pl}}} \gtrsim \frac{M}{V\left(\phi_{+}\right)^{\frac{1}{4}}} .
$$

При этом неравенство (1) вполне может удовлетворяться, а плотность энергии $V\left(\phi_{+}\right)$ может быть мала по сравнению с планковской. Таким образом, в некоторой области параметров модели приближение внешней метрики законно, и в то же время гравитационными эффектами пренебрегать нельзя.

В дальнейшем мы будем изучать скалярное поле во внешней метрике, поэтому можем произвольным образом сдвинуть начало отсчета его энергии. Удобно положить $V(0)=0$, как изображено на рис. 2 .

Выбор состояния квантовых возбуждений над ложным классическим вакуумом тесно связан с выбором системы отсчета. Мы будем использовать систему с плоскими поверхностями постоянного времени, в которой метрика де Ситтера имеет вид

$$
d l^{2}=\frac{1}{H^{2} \eta^{2}}\left(d \eta^{2}-d x_{i} d x_{i}\right)
$$

Конформное время $\eta$ изменяется от $-\infty$ до 0 и связано с синхронным временем $t$ как

$$
\eta=-\frac{1}{H} e^{-H t}
$$




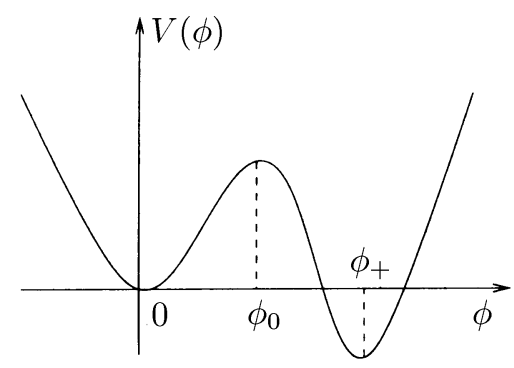

Рис. 2

Эта система координат покрывает только половину пространства де Ситтера (см., например, [13]), однако лишь эта половина представляет интерес для инфляционных моделей. Действие модели в таких координатах имеет вид

$$
S=\int \frac{d^{3} x d \eta}{H^{4} \eta^{4}}\left[\frac{H^{2} \eta^{2}}{2}\left(\left(\frac{\partial \phi}{\partial \eta}\right)^{2}-\left(\frac{\partial \phi}{\partial x^{i}}\right)^{2}\right)-V(\phi)\right] .
$$

Произведем разложение малых отклонений поля от ложного вакуума по естественной для выбранных координат системе полевых мод [9]:

$$
\begin{gathered}
\hat{\phi}(\mathbf{x}, \eta)=\int \frac{d^{3} k}{(2 \pi)^{\frac{3}{2}}} H \eta\left(a_{k} \chi_{k}(\eta)+a_{-k}^{+} \chi_{k}^{*}(\eta)\right) e^{i \mathbf{k x}}, \\
\chi_{k}(\eta)=\frac{(\pi \eta)^{\frac{1}{2}}}{2} H_{\nu}^{(2)}(k \eta), \quad \nu^{2}=\frac{9}{4}-\frac{m^{2}}{H^{2}}, \quad m^{2}=V^{\prime \prime}(0), \quad k=|\mathbf{k}| .
\end{gathered}
$$

Здесь $H_{\nu}$ - функция Ганкеля. Конформный квантовый вакуум над ложным классическим вакуумом определяется как состояние, уничтожаемое всеми операторами $a_{k}$ : $a_{k}|0\rangle=0$. Хотя это определение имеет смысл только в теории возмущений, для нас оно будет вполне достаточно. Наш выбор системы координат и вакуума обусловлен несколькими причинами:

1. Такая система координат и такое определение вакуума наиболее естественно возникают в инфляционных теориях (см., например, [14]).

2. Выбранный вакуум инвариантен относительно пространственных трансляций, $x \rightarrow x+a$, и масштабных преобразований, $\eta \rightarrow \alpha \eta, x \rightarrow \alpha x$, являюшихся глобальными изометриями рассматриваемой части пространства де Ситтера.

3. Полевые моды $\chi_{k}$ сводятся к отрицательно-частотным плоским волнам при больших отрицательных $\eta$ :

$$
\chi_{k} \rightarrow \frac{1}{(2 k)^{\frac{1}{2}}} e^{-i k \eta}, \quad \eta \rightarrow \infty .
$$

Поэтому можно ожидать, что при больших отрицательных временах теория в координатах $(\eta, x)$ будет похожа на теорию в плоском пространстве, и это действительно имеет место (см. ниже).

Заданное таким образом состояние ложного вакуума метастабильно. Наша задача найти показатель главной квазиклассической экспоненты для вероятности его распада. 


\section{3. КЛАССИЧЕСКОЕ РЕШЕНИЕ И ГРАНИЧНЫЕ УСЛОВИЯ}

В этом разделе мы покажем, что в квазиклассическом случае показатель экспоненты для вероятности распада ложного вакуума действительно определяется некоторым классическим решением уравнений скалярного поля, и сформулируем граничные условия, накладываемые на это решение.

Введем следуюшие обозначения: $|n, \eta\rangle$ - состояние $n$ в момент времени $\eta$ (мы работаем в гейзенберговском представлении); так, $|0, \eta\rangle$ - состояние ложного вакуума в момент $\eta$, а $|\phi(\mathbf{x}), \eta\rangle$ - собственное состояние полевых операторов, взятых в момент $\eta$. Для вычисления вероятности распада ложного вакуума нам необходим матричный элемент $\left\langle\phi_{f}\left(\mathbf{x}, \eta_{f}\right) \mid 0, \eta_{i}\right\rangle$, где $\eta_{f}$ и $\eta_{i}-$ конечный и начальный моменты времени. Он может быть представлен в виде функционального интеграла

$$
\left\langle\phi_{f}\left(\mathbf{x}, \eta_{f}\right) \mid 0, \eta_{i}\right\rangle=\int D\left[\phi_{i}(\mathbf{x})\right]\left\langle\phi_{i}(\mathbf{x}), \eta_{i} \mid 0, \eta_{i}\right\rangle \int D[\phi(\mathbf{x}, \eta)] e^{i S[\phi]} .
$$

Действие $S[\phi]$ в показателе экспоненты в (5) берется за промежуток времени $\left(\eta_{i}, \eta_{f}\right)$ на полевой конфигурации $\phi(\mathbf{x}, \eta)$, удовлетворяюшей граничным условиям

$$
\phi\left(\mathbf{x}, \eta_{i}\right)=\phi_{i}(\mathbf{x}), \quad \phi\left(\mathbf{x}, \eta_{f}\right)=\phi_{f}(\mathbf{x}) .
$$

Внешнее интегрирование производится по начальным конфигурациям поля. Сделаем следуюшие два замечания:

1. Интервал времени $\left(\eta_{i}, \eta_{f}\right)$ предполагается большим по сравнению с наибольшим характерным временным масштабом теории. В конечном итоге нас интересует предел $\eta_{i} \rightarrow-\infty$.

2. До сих пор переменные х и $\eta$ имели смысл действительных координат в пространстве де Ситтера. Однако можно рассматривать пространство комплексных переменных $(\mathbf{x}, \eta)$ и аналитические продолжения метрики и полей в это пространство. При этом четырехмерная гиперповерхность, по которой производится интегрирование при вычислении действия в (5), может быть произвольным образом деформирована в этом комплексном пространстве при условии фиксации "граничных точек", т.е. трехмерных начальной и конечной поверхностей.

Для ширины распада ложного вакуума имеем

$$
\Gamma=\int D\left[\phi_{f}(\mathbf{x})\right]\left|\left\langle\phi_{f}, \eta_{f} \mid 0, \eta_{i}\right\rangle\right|^{2},
$$

где интегрирование производится по конфигурациям $\phi_{f}(\mathbf{x})$, близким к истинному вакууму (смысл последнего условия станет ясен ниже). Подставляя (5) в (6), получаем

$$
\begin{aligned}
\Gamma= & \int D\left[\phi_{f}(\mathbf{x})\right] \int D\left[\phi_{1 i}(\mathbf{x})\right] D\left[\phi_{2 i}^{*}(\mathbf{x})\right]\left\langle\phi_{1 i}, \eta_{i} \mid 0, \eta_{i}\right\rangle\left\langle\phi_{2 i}, \eta_{i} \mid 0, \eta_{i}\right\rangle^{*} \times \\
& \times \int D\left[\phi_{1}(\mathbf{x}, \eta)\right] D\left[\phi_{2}(\mathbf{x}, \eta)\right] e^{i\left(S\left[\phi_{1}\right]-S^{*}\left[\phi_{2}\right]\right)} .
\end{aligned}
$$

Нашей ближайшей целью является последовательное вычисление входяших в (7) функциональных интегралов методом перевала. (Применение метода перевала предполагает, конечно, квазиклассичность рассматриваемой задачи.) Проинтегрируем сначала по 
$D\left[\phi_{1}\right] D\left[\phi_{2}\right]$. Седловая точка подынтегрального выражения задается решениями классических уравнений поля. Соответствуюшие классические решения $\phi_{1}(\mathbf{x}, \eta)$ и $\phi_{2}(\mathbf{x}, \eta)$ удовлетворяют условиям

$$
\phi_{1}\left(\mathbf{x}, \eta_{i}\right)=\phi_{1 i}(\mathbf{x}), \quad \phi_{2}\left(\mathbf{x}, \eta_{i}\right)=\phi_{2 i}(\mathbf{x}), \quad \phi_{1}\left(\mathbf{x}, \eta_{f}\right)=\phi_{2}\left(\mathbf{x}, \eta_{f}\right)=\phi_{f}(\mathbf{x}) .
$$

Чтобы произвести интегрирование по $D\left[\phi_{1 i}\right] D\left[\phi_{2 i}\right]$, необходимо знать явньй вид матричных элементов типа $\left\langle\phi_{i}(\mathbf{x}), \eta_{i} \mid 0, \eta_{i}\right\rangle$. Их вычисление практически совпадает с соответствующим вычислением в случае плоского пространства (это обусловлено стремлением полевых мод $\chi_{k}$ к плоским волнам при $\left.\eta \rightarrow-\infty\right)$. Так как рассматриваемые матричные элементы заметно отличны от нуля лишь для конфигураций поля, достаточно близких к ложному вакууму, можно пользоваться теорией возмушений. Введем сопряженные полевым операторам импульсы

$$
\begin{aligned}
\hat{\pi}(\mathbf{x}, \eta)= & \frac{1}{H^{2} \eta^{2}} \frac{\partial \hat{\phi}}{\partial \eta}=\int \frac{d^{3} k}{(2 \pi)^{\frac{3}{2}}} \frac{1}{H^{2} \eta^{2}}\left(a_{k} \chi_{k}(\eta)+a_{-k}^{+} \chi_{k}^{*}(\eta)\right) e^{i \mathbf{k} \mathbf{x}}+ \\
& +\int \frac{d^{3} k}{(2 \pi)^{\frac{3}{2}}} \frac{1}{H \eta}\left(a_{k} \dot{\chi}_{k}(\eta)+a_{-k}^{+} \dot{\chi}_{k}^{*}(\eta)\right) e^{i \mathbf{k x}} .
\end{aligned}
$$

Замечая, что при больших отрицательных $\eta$ первым слагаемым в (9) можно пренебречь, и переходя к пространственным фурье-компонентам

$$
\hat{\phi}\left(\mathbf{k}, \eta_{i}\right)=H \eta_{i}\left(a_{k} \chi_{k}\left(\eta_{i}\right)+a_{-k}^{+} \chi_{k}^{*}\left(\eta_{i}\right)\right), \quad \hat{\pi}\left(\mathbf{k}, \eta_{i}\right)=\frac{1}{H \eta}\left(a_{k} \dot{\chi}_{k}\left(\eta_{i}\right)+a_{-k}^{+} \dot{\chi}_{k}^{*}\left(\eta_{i}\right)\right),
$$

получаем следующие коммутационные соотношения:

$$
\left[\hat{\pi}\left(-\mathbf{k}, \eta_{i}\right), \hat{\phi}\left(\mathbf{q}, \eta_{i}\right)\right]=-i \delta(\mathbf{k}-\mathbf{q})
$$

Следовательно, операторы $\hat{\phi}\left(\mathbf{k}, \eta_{i}\right)$ и $\hat{\pi}\left(\mathbf{k}, \eta_{i}\right)$ могут быть реализованы как умножение на функцию $\phi(\mathbf{k})$ и взятие вариационной производной $-i \delta / \delta \phi(-\mathbf{k})$, соответственно. Таким образом, мы приходим к координатному представлению. Состояние $\left|0, \eta_{i}\right\rangle$ в этом представлении является функционалом $F[\phi(\mathbf{k})]=\left\langle\phi(\mathbf{k}), \eta_{i} \mid 0, \eta_{i}\right\rangle$. Уравнение для него мы получим, выражая операторы $a_{k}$ через $\hat{\phi}\left(\mathbf{k}, \eta_{i}\right)$ и $\hat{\pi}\left(\mathbf{k}, \eta_{i}\right)$ и вспоминая, что $a_{k}$ аннигилируют вакуум:

$$
\left(\frac{\delta}{\delta \pi(-\mathbf{k})}+\frac{k}{\left(H \eta_{i}\right)^{2}} \phi(\mathbf{k})\right) F=0
$$

(при получении (10) использована асимптотика (4)). Отсюда имеем

$$
F=F_{0} \exp \left[-\int \frac{k \phi(\mathbf{k}) \phi(-\mathbf{k})}{2\left(H \eta_{i}\right)^{2}} d^{3} k\right] .
$$

Константа $F_{0}$ определяется из условия нормировки и для нас несушественна.

Подставим (11) в (7) и проинтегрируем по $D\left[\phi_{1 i}\right]$. Седловая точка подынтегрального выражения определяется условием

$$
\frac{\delta}{\delta \phi_{1 i}(-\mathbf{k})}\left(-\int \frac{k \phi_{1 i}(\mathbf{k}) \phi_{1 i}(-\mathbf{k})}{2\left(H \eta_{i}\right)^{2}} d^{3} k+i S\left[\phi_{1}\right]\right)=0,
$$


из которого следует, что

$$
\frac{k}{\left(H \eta_{i}\right)^{2}} \phi_{1 i}(\mathbf{k})+i \frac{1}{\left(H \eta_{i}\right)^{2}} \dot{\phi}_{1}\left(\mathbf{k}, \eta_{i}\right)=0 .
$$

Таким образом, мы приходим к условию положительной частотности при $\eta \rightarrow-\infty$ классического решения $\phi_{1}$. Аналогичные рассуждения приводят к точно такому же условию для $\phi_{2}$. Граничные условия при $\eta_{f}$ для $\phi_{1}$ и $\phi_{2}$ тоже одинаковы (см. (8)), кроме того, $\phi_{1}$ и $\phi_{2}$ являются решениями одних и тех же классических уравнений. Следовательно, $\phi_{1}$ и $\phi_{2}$ - одна и та же функция ${ }^{2)}$, которую мы обозначим просто $\phi$. Итак, седловое интегрирование по промежуточным и начальным значениям поля дает

$$
\Gamma \propto \int D\left[\phi_{f}\right] e^{i\left(S[\phi]-S^{*}[\phi]\right)},
$$

где $\phi(\mathbf{x}, \eta)$ - решение (вообше говоря, комплексное) классического уравнения поля во внешней метрике де Ситтера, удовлетворяющее граничным условиям

$$
\phi\left(\mathbf{x}, \eta_{f}\right)=\phi_{f}(\mathbf{x})
$$

$\phi$ положительно-частотно при $\eta \rightarrow-\infty$.

Поскольку нас в этой работе интересует лишь главная квазиклассическая экспонента, предэкспоненциальный множитель в (12) не выписан. Отметим, что граничное условие (13), как и в случае пространства Минковского, позволяет не только произвольным образом деформировать контур интегрирования, но и смешать граничную точку $\eta_{i}=-\infty$ в верхней полуплоскости комплексной переменной $\eta$. Контуром интегрирования (или просто контуром) мы для краткости назьваем гиперповерхность, по которой производится интегрирование при вычислении действия в (12).

В (12) осталось еще одно интегрирование. Находим седловое уравнение

$$
\dot{\phi}\left(\mathbf{x}, \eta_{f}\right)-\dot{\phi}^{*}\left(\mathbf{x}, \eta_{f}\right)=0
$$

что вместе с требованием действительности $\phi_{f}(\mathbf{x})$ дает следуюшее условие:

$$
\phi(\mathbf{x}, \eta) \text { действительно при } \eta>\eta_{f} \text {. }
$$

При продолжении решения $\phi(\eta)$ (мы временно отвлечемся от пространственной зависимости поля $\phi)$ из области $\eta>\eta_{f}$ вдоль действительной оси в область $\eta<\eta_{f}$ оно будет оставаться действительным. Может показаться, что это противоречит условию (13). Однако это говорит лишь о том, что решения, обладаюшего требуемыми свойствами, не сушествует на действительной оси и его следует искать на деформированном контуре, огибаюшем особые точки функции $\phi[15]$.

Значения $\phi$ в верхней и нижней полуплоскостях связаны равенством $\phi\left(\eta^{*}\right)=\phi^{*}(\eta)$. Учитывая это, преобразуем выражение, стояшее в показателе экспоненты в (12):

$$
i\left(S[\phi]-S^{*}[\phi]\right)=i\left(S_{C}[\phi]-S_{C^{*}}[\phi]\right)=i S_{C+C^{*}}[\phi] .
$$

\footnotetext{
2) Действительно, общее решение уравнений второго порядка содержит две произвольные комплексные функции трех переменных. Условие положительной частотности уничтожает одну из этих степеней свободы. Вторая же функция определяется из граничного условия в конечный момент времени.
} 


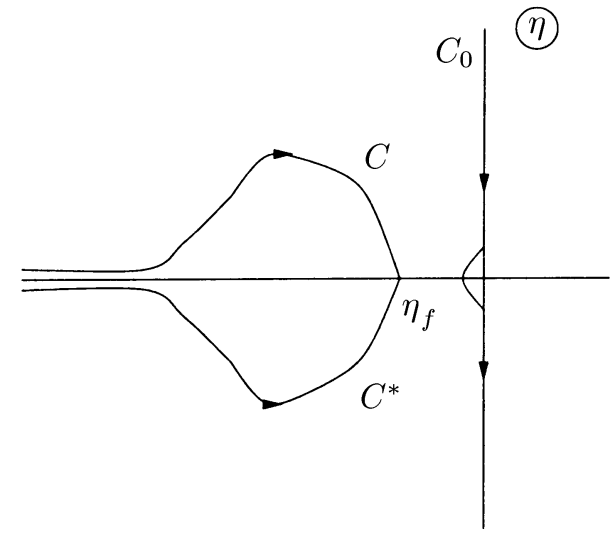

Рис. 3

Здесь через $S_{C}, S_{C}$ и $S_{C+C^{*}}$ обозначено действие, вычисленное соответственно на контуре $C$, “сопряженном" ему контуре $C^{*}$ и их объединении (см. рис. 3 ). Теперь мы можем оторвать контур интегрирования от точки $\eta_{f}$, к которой он был до сих пор привязан, и деформировать его в контур $C_{0}$, состоящий из мнимой оси и бесконечно малой полуокружности, огибаюшей особую точку метрики $\eta=0$.

Удобно сделать замену $\eta=i \zeta$ и преобразовать действие к виду

$$
i S_{C_{0}}[\phi]=\int d^{3} x \int_{-\infty}^{\infty} d \zeta \frac{1}{H^{4} \zeta^{4}}\left[\frac{H^{2} \zeta^{2}}{2}\left(\left(\frac{\partial \phi}{\partial \zeta}\right)^{2}+\left(\frac{\partial \phi}{\partial x^{i}}\right)^{2}\right)-V(\phi)\right] \equiv-S_{\zeta}[\phi] .
$$

(Штрих у знака интеграла указывает на необходимость правильного обхода нуля.) Подведем итоги этого раздела:

1. Для главной квазиклассической экспоненты распада ложного вакуума получено выражение

$$
\Gamma \propto e^{-S_{\zeta}[\phi]} .
$$

2. Функция $\phi$ в (16) представляет собой классическое решение уравнений поля, взятое на контуре $C_{0}$.

3. Аналитическое продолжение $\phi$ на действительную ось $\eta$ действительно.

4. $\phi(\zeta)$ стремится к нулю при $\zeta \rightarrow \infty$ (последнее свойство есть следствие условия (13)).

Для дальнейшего преобразования “контура интегрирования" (т.е. четырехмерной гиперповерхности в пространстве комплексных $\mathbf{x}$ и $\eta$ ) полезно изучить аналитические продолжения метрики де Ситтера.

\section{4. АНАЛИТИЧЕСКОЕ ПРОДОЛЖЕНИЕ МЕТРИКИ}

В предыдушем разделе мы ввели координату $\zeta$, связанную с $\eta$ как $\eta=i \zeta$. Изучим сначала метрику пространства, задаваемого координатами $(\mathbf{x}, \zeta)$ при $0<\zeta<\infty$. Из (2) очевидно, что элемент длины равен

$$
d l^{2}=\frac{d \zeta^{2}+d x_{i}^{2}}{H^{2} \zeta^{2}},
$$


т.е. метрика имеет евклидову сигнатуру. Это пространство обладает постоянной отрицательной кривизной, что непосредственно следует из аналогичного свойства пространства де Ситтера [9]. Кроме того, можно показать, что в отличие от рассматривавшейся половины пространства де Ситтера оно геодезически полно.

Введем сферические координаты в трехмерном пространстве с координатами $x_{i}$, тогда (17) перепишется в виде

$$
d l^{2}=\frac{d \zeta^{2}+d \rho^{2}+\left(d \Omega_{2}\right)^{2}}{H^{2} \zeta^{2}}
$$

где $\rho=\sqrt{x_{1}^{2}+x_{2}^{2}+x_{3}^{2}}$, а $\left(d \Omega_{2}\right)^{2}=d \theta^{2}+\sin \theta^{2} d \varphi^{2}, 0 \leqslant \theta \leqslant \pi, 0 \leqslant \varphi<2 \pi$, - элемент длины на единичной двумерной сфере. До сих пор величины $x, \eta, \zeta, \rho$ в нашем рассмотрении имели размерность длины. Для дальнейшего, однако, удобно их обезразмерить, а именно, измерять в единицах обратной постоянной Хаббла. При этом выражение для элемента длины останется прежним, а для действия лишь слегка поменяет вид:

$$
S_{\zeta}=-\int d^{3} x \int_{-\infty}^{\infty} d \zeta \frac{1}{\zeta^{4}}\left[\frac{\zeta^{2}}{2 H^{2}}\left(\left(\frac{\partial \phi}{\partial \zeta}\right)^{2}+\left(\frac{\partial \phi}{\partial x^{i}}\right)^{2}\right)-\frac{V(\phi)}{H^{4}}\right] .
$$

Введем во всем рассматриваемом пространстве координаты, в определенном смысле подобные сферическим. А именно, выберем точку - начало отсчета с $\rho=0, \zeta=\zeta_{0}$ и выпустим из нее всевозможные геодезические. Расстояние вдоль этих геодезических и будет нашей радиальной координатой $s$. Конкретнее,

$$
s=\int_{\left(0, \zeta_{0}\right)}^{(\rho, \zeta)} d l=\int_{\left(0, \zeta_{0}\right)}^{(\rho, \zeta)} \frac{d \zeta^{\prime}}{\zeta^{\prime}} \sqrt{1+\left(\frac{d \rho}{d \zeta^{\prime}}\right)^{2}} .
$$

Интегрирование производится вдоль геодезической, соединяющей точки $(\rho, \zeta)$ и $(0, \zeta)$, в аналогичном смысле понимается и производная $d \rho / d \zeta^{\prime}$. Заметив, что изменение $\zeta_{0}$ эквивалентно изменению масштаба и ни на что, по сушеству, не влияет, положим его в дальнейшем для определенности равным единице. После очевидных, но достаточно громоздких выкладок, которые мы опускаем, приходим к следующему выражению координат $\rho$ и $\zeta$ через $s$ и угловую переменную $\psi$, характеризующую направление соответствуюшей геодезической:

$$
\zeta=\frac{1}{\operatorname{ch} s-\cos \psi \operatorname{sh} s}, \quad \rho=\frac{\sin \psi \operatorname{sh} s}{\operatorname{ch} s-\cos \psi \operatorname{sh} s}, \quad 0 \leqslant s<\infty, \quad 0 \leqslant \psi \leqslant \pi .
$$

Линии постоянных $s$ и $\psi$ (дуги окружности) изображены на рис. 4.

Элемент длины в новых координатах имеет следующий вид:

$$
d l^{2}=\frac{1}{H^{2}}\left(d s^{2}+\operatorname{sh}^{2} s\left(d \psi^{2}+\sin \psi^{2}\left(d \Omega_{2}\right)^{2}\right)\right) .
$$

Обсудим замену координат (20). Уравнение $\psi=0$ описывает на плоскости $(\rho, \zeta)$ луч $\rho=0,1 \leqslant \zeta<\infty$, а уравнение $\psi=\pi$ - отрезок $\rho=0,0<\zeta \leqslant 1$. При $\psi=\pi / 2$ имеем дугу окружности $\rho^{2}+\zeta^{2}=1$. Если, зафиксировав $\psi \neq 0$, устремить $s$ к бесконечности, то получим

$$
\zeta \sim \frac{e^{-s}}{1-\cos \psi}, \quad \rho \rightarrow \frac{\sin \psi}{1-\cos \psi}, \quad s \rightarrow \infty
$$




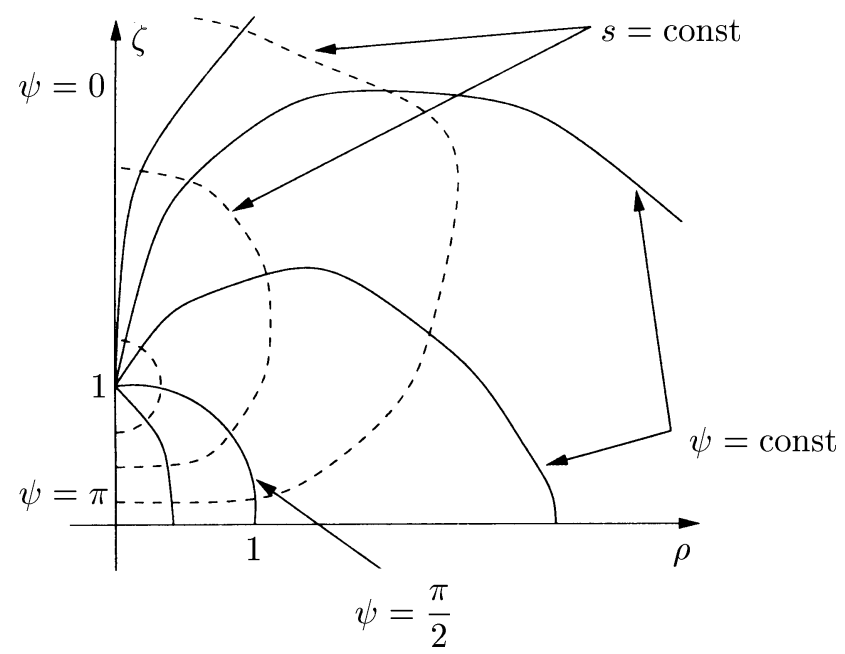

Рис. 4

Наконец, асимптотика $s \rightarrow \infty, \psi \sim e^{-2 s}$ соответствует пределам $\rho \rightarrow$ const, $\zeta \rightarrow \infty$.

Рассмотрим теперь $s$ как комплексную переменную (оставляя $\psi$ действительным) и изучим, каким аналитическим продолжениям метрики это соответствует. Для нас интересны следующие случаи:

1. $s=-i \pi+\hat{s}$, где $\hat{s}$ действительно. При подстановке этой замены в $(20)$ получим, что $\rho$ выражается через $\hat{s}$ так же, как оно выражалось через $s$, а формула для $\zeta$ отличается лишь знаком. Следовательно, такие значения $s$ описывают пространство с отрицательными $\zeta$; структура этого пространства полностью аналогична структуре пространства с положительными $\zeta$.

2. $s=-i(\pi / 2)+s^{\prime}$, где $s^{\prime}$ действительно. Такая подстановка приводит к следующим выражениям для $\rho$ и $\zeta$ :

$$
\rho=\frac{\sin \psi \operatorname{ch} s^{\prime}}{\operatorname{sh} s^{\prime}-\cos \psi \operatorname{ch} s^{\prime}}, \quad \zeta=\frac{i}{\operatorname{sh} s^{\prime}-\cos \psi \operatorname{ch} s^{\prime}} .
$$

При ограничении $\cos \psi<$ th $s^{\prime}$ формулы (23) описывают пространство с $\rho>0$ и чисто мнимым $\zeta$, изменяюшимся от 0 до $+i \infty$. Это пространство есть не что иное, как половина пространства де Ситтера, рассматривавшаяся в разделах 2 и 3 (ограничение на изменение $s^{\prime}$ при фиксированном $\psi$ связано, конечно, с его геодезической неполнотой). Линии постоянных $s^{\prime}, \psi$ на плоскости $\eta=i \zeta, \rho$ имеют вид, изображенный на рис. 5 . Это пространство характеризуется метрикой

$$
d l^{2}=\frac{1}{H^{2}}\left(d s^{\prime 2}-\operatorname{ch}^{2} s^{\prime}\left(d \psi^{2}+\sin ^{2} \psi\left(d \Omega_{2}\right)^{2}\right)\right)
$$

3. $s=-i \sigma, \sigma$ действительно и принадлежит отрезку $[0, \pi]$. При такой замене метрика (21) переходит в следующую:

$$
d l^{2}=\frac{1}{H^{2}}\left(-d \sigma-\sin ^{2} \sigma\left(d \psi^{2}+\sin ^{2} \psi\left(d \Omega_{2}\right)^{2}\right)\right),
$$






Рис. 5

что с точностью до знака совпадает с метрикой на четырехмерной сфере.

\section{5. РЕШЕНИЕ КОУЛМЕНА-ДЕ ЛЮЧЧИА}

В приближении внешней (нединамической) метрики конфигурация Коулменаде Люччиа представляет собой решение уравнения скалярного поля на четырехмерной сфере. В этом разделе мы убедимся, что эта конфигурация действительно является решением граничной задачи, сформулированной в разделе 3 . Перейдем от интегрирования по $d x d \zeta$ в действии (19) к интегрированию по четырехмерной сфере. Интеграл, входящий в (19), может быть представлен в виде

$$
S_{\zeta}=-\int_{0}^{\infty} \rho^{2} d \rho \int_{-\infty}^{\infty} \frac{d \zeta}{\zeta^{4}} f(\rho, \zeta)
$$

где $f(\rho, \zeta)$ - аналитическая функция двух переменных. Разобьем его на три слагаемых: интегралы по положительным и отрицательным $\zeta$ и интеграл вблизи особой точки $\zeta=$ 0. В первом из них сделаем замену переменных:

$$
\int_{0}^{\infty} \rho^{2} d \rho \int_{0}^{\infty} \frac{d \zeta}{\zeta^{4}} f(\rho, \zeta)=\int_{0}^{\pi} d \psi \int_{0}^{\infty} d s \operatorname{sh}^{3} s \sin ^{2} \psi \tilde{f}(\psi, s),
$$

где

$$
\tilde{f}(\psi, s)=f\left(\frac{\sin \psi \operatorname{sh} s}{\operatorname{ch} s-\cos \psi \operatorname{sh} s}, \frac{1}{\operatorname{ch} s-\cos \psi \operatorname{sh} s}\right) .
$$

Аналогично поступим и со вторым слагаемым:

$$
\begin{aligned}
& \int_{0}^{\infty} \rho^{2} d \rho \int_{-\infty}^{0} \frac{d \zeta}{\zeta^{4}} f(\rho, \zeta)= \\
& \quad=\int_{0}^{\pi} d \psi \int_{0}^{\infty} d \hat{s} \operatorname{sh}^{3} \hat{s} \sin ^{2} \psi f\left(\frac{\sin \psi \operatorname{sh} \hat{s}}{\operatorname{ch} \hat{s}-\cos \psi \operatorname{sh} \hat{s}},-\frac{1}{\operatorname{ch} \hat{s}-\cos \psi \operatorname{sh} \hat{s}}\right)
\end{aligned}
$$


Перепишем последнее выражение следующим образом (см. раздел 4):

$$
-\int_{0}^{\pi} d \psi \int_{-i \pi}^{-i \pi+\infty} d s \operatorname{sh}^{3} s \sin ^{2} \psi \tilde{f}(\psi, s) .
$$

Итак, интегрирование по положительным и отрицательным $\zeta$ соответствует в комплексной плоскости $s$ интегрированию по лучам $[0, \infty)$ и $(\infty-i \pi,-i \pi]$, соответственно, от одной и той же аналитической функции $\tilde{f}$. Чтобы получить полный интеграл $(26)$, контур нужно замкнуть. Из (22) видно, что $\zeta$ мало, когда $s$ велико, и движение по отрезку $\left[s_{1}, s_{1}+i \pi\right]$ при большом действительном $s_{1}$ соответствует обходу особенности $\zeta=0$ в верхней полуплоскости, т.е. именно так, как подразумевается в интеграле (26). Таким образом, контур нужно замыкать на бесконечности, и действие $S_{\zeta}$ равно следуюшему выражению:

$$
S_{\zeta}=-\int_{0}^{\pi} d \psi \int_{C} d s \operatorname{sh}^{3} s \sin ^{2} \psi \tilde{f}(\psi, s),
$$

где контур $C$ изображен на рис. 6.

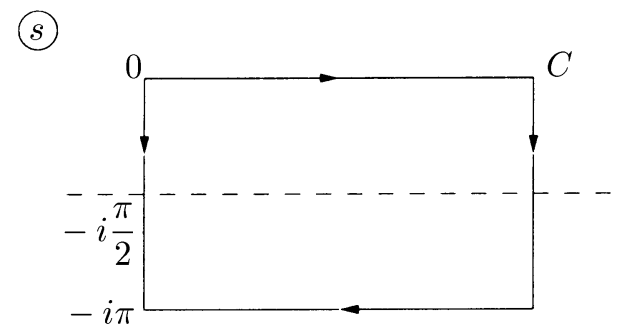

Рис. 6

Этот контур можно продеформировать в отрезок мнимой оси, соединяющий точки 0 и $-i \pi$. Действие при этом, как нам и хотелось, принимает вид интеграла по сфере:

$$
S_{\zeta}=-\int_{0}^{\pi} d \psi \int_{0}^{\pi} d \sigma \sin ^{3} \sigma \sin ^{2} \psi \tilde{f}(\psi,-i \sigma)
$$

Для завершения перехода на сферу нам нужно сформулировать на ней уравнения поля и переписать накладываемые на классическое решение условия (см. раздел 3) в более удобном виде. Первая задача решается легко: достаточно лишь выписать явное выражение для функции $\tilde{f}$ из (28), получающееся заменой переменных в исходной формуле (19):

$$
\tilde{f}(\psi,-i \sigma)=-\int_{0}^{\pi} d \theta \sin \theta \int_{0}^{2 \pi} d \varphi\left(\frac{\left(\nabla_{S^{4}} \phi\right)^{2}}{2 H^{2}}+\frac{V(\phi)}{H^{4}}\right),
$$

где через $\nabla_{S^{4}}$ обозначен градиент на поверхности 4-сферы. (Заметим, что за счет появления обшего знака минус перед интегралом (29) градиентный и потенциальный члены входят в него с одинаковым знаком, т.е. произошло переворачивание скалярного потенциала (ср. с $[2,3,5])$.) Теперь варьированием действия получаем уравнения

$$
\Delta_{S^{4}} \phi=\frac{V^{\prime}(\phi)}{H^{2}},
$$


где $\Delta_{S^{4}}$ - оператор Лапласа на 4-сфере.

В терминах решения на сфере просто формулируется и условие действительности $\phi$ при действительных $\eta$ (см. п. 3 в конце раздела 3 ). Оно эквивалентно требованию действительности $\phi$ при

$$
s=-i \frac{\pi}{2}+s^{\prime},
$$

где $s^{\prime}$ действительно (см. раздел 4). Последнее приводит к соотношению

$$
\left.\phi\right|_{s=z-i \frac{\pi}{2}}=\left.\phi^{*}\right|_{s=z^{*}-i \frac{\pi}{2}} .
$$

Сделаем сушественное предположение: решение $\phi$ на сфере, т.е. при $s=-i \sigma$, действительно. Это предположение вместе с (31) дает следующие условия:

УСловиЕ 1. Для того чтобы решение $\phi$ было действительным при действительных $\eta$, необходимо и достаточно, чтобы $\phi$ на сфере было симметрично относительно "экватора" $\sigma=\pi / 2$.

Требование же убывание $\phi$ при стремлении $\zeta$ к $\infty$ (см. п. 4 в конце раздела 3$)$ никак не переносится на сферу, поэтому его приходится формулировать следуюшим образом:

УСловиЕ 2. Аналитическое продолжение полученного на сфере решения классических уравнений поля $(30)$ в область действительных $s$ стремится к нулю при устремлении $s$ к бесконечности, а $\psi$ - к нулю как $\psi \sim e^{-2 s}$.

Для построения решения введем на сфере новые угловые переменные $\sigma^{\prime}, \psi^{\prime}$, связанные $\mathrm{c} \sigma, \psi$ соотношениями

$$
\cos \sigma^{\prime}=\sin \sigma \cos \psi, \quad \sin \sigma^{\prime} \cos \psi^{\prime}=\cos \sigma .
$$

Такая замена соответствует переносу полюса системы координат в точку $(\sigma=\pi / 2$, $\psi=0)$. В новых координатах решение $\phi$ должно быть симметрично относительно "меридиана" $\psi^{\prime}=\pi / 2$. Предположим, следуя Коулмену и де Люччиа, что $\phi$ обладает большей симметрией, а именно, что оно $O(4)$-симметрично. Тогда $\phi$ зависит только от одной переменной $\sigma^{\prime}$. Пользуясь этим, упрошаем выражение для действия:

$$
S_{\zeta}=2 \pi^{2} \int_{0}^{\pi} d \sigma^{\prime} \sin ^{3} \sigma^{\prime}\left(\frac{1}{2 H^{2}}\left(\frac{d \phi}{d \sigma^{\prime}}\right)^{2}+\frac{V(\phi)}{H^{4}}\right),
$$

из которого получаем уравнение для $\phi$ :

$$
\frac{d^{2} \phi}{d \sigma^{\prime 2}}+\frac{3}{\operatorname{tg} \sigma^{\prime}} \frac{d \phi}{d \sigma^{\prime}}-\frac{V^{\prime}(\phi)}{H^{2}}=0 .
$$

Дополнительные условия, которые необходимо наложить на $\phi$, таковы:

a)

$$
\left.\frac{d \phi}{d \sigma^{\prime}}\right|_{\sigma^{\prime}=0}=\left.\frac{d \phi}{d \sigma^{\prime}}\right|_{\sigma^{\prime}=\pi}=0
$$

(следует из требования гладкости $\phi$ );

б) $\phi$ не постоянно (это необходимо для выполнения условия 2 (см. ниже)); 
в) $\phi\left(\sigma^{\prime}=0\right)$ близко к ложному вакууму, т.е. к $\phi=0 ; \phi\left(\sigma^{\prime}=\pi\right)$ близко к истинному вакууму, т.е. к $\phi_{+}$.

Решение, обладаюшее перечисленными свойствами, существует для широкого класса потенциалов $V(\phi)$, для которых (см. $[5,7,12])$

$$
\left|\frac{V^{\prime \prime}\left(\phi_{0}\right)}{H^{2}}\right|>4 \text {. }
$$

Если неравенство (36) не выполняется, условию 2 удовлетворить не удается [ $[7,12]$. Этот случай будет рассмотрен в следуюшем разделе, а пока будем предполагать, что реализуется первая возможность. Построенное решение автоматически удовлетворяет условию 1 в силу $O(4)$-симметрии, проверку же условия 2 мы пока отложим и исследуем продолжение $\phi$ в пространство действительных $\eta$.

На этом этапе удобнее считать, что $\phi$ зависит не от $\sigma^{\prime}$, а от связанной с ней переменной $\mu=\cos \sigma^{\prime}$, которая согласно (32) следуюшим образом выражается через введенные в разделе 4 координаты:

$$
\mu=\sin \sigma \cos \psi=i \operatorname{sh} s \cos \psi=\operatorname{ch} s^{\prime} \cos \psi .
$$

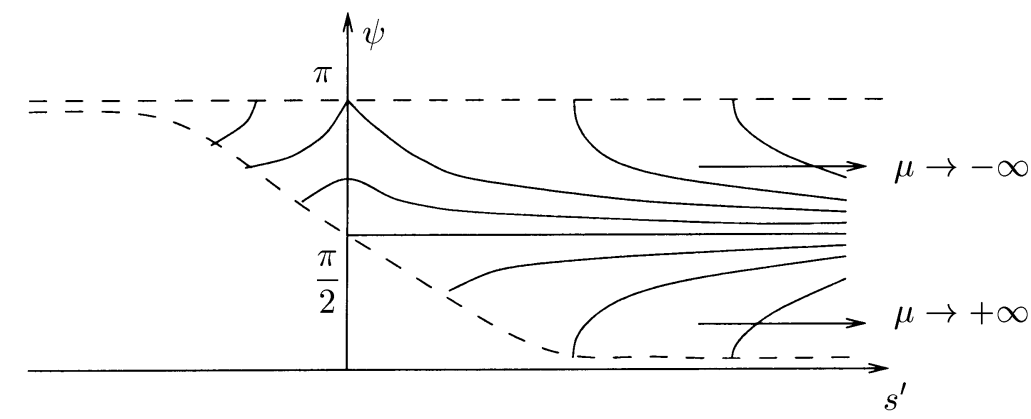

Рис. 7

На рис. 7 показаны линии уровня $\mu$ в координатах $\left(s^{\prime}, \psi\right)$ (изображена только область $\cos \psi<$ th $s$, соответствуюшая физическому пространству де Ситтера). Соотнося рис. 7 и 5 , получаем картину линий уровня $\mu$, а следовательно, и $\phi$ на плоскости

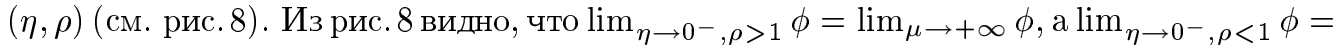
$\lim _{\mu \rightarrow+\infty} \phi$. Таким образом, для лучшего понимания поведения $\phi$ нужно изучить его асимптотики при $\mu \rightarrow \pm \infty$.

Стремление $\mu \mathrm{k}+\infty$ эквивалентно следуюшему поведению $\sigma^{\prime}: \sigma^{\prime}=i \sigma^{\prime \prime}$, где $\sigma^{\prime \prime}$ действительно и стремится к $+\infty$. При $\mu \rightarrow-\infty$ значение $\sigma^{\prime}$ равно $\pi+i \sigma^{\prime \prime}$, где $\sigma^{\prime \prime}$ снова действительно и стремится к $+\infty$. Уравнение для поля $\phi$ в этих областях получается аналитическим продолжением уравнения (34):

$$
\frac{d^{2} \phi}{d \sigma^{\prime \prime 2}}+\frac{3}{\operatorname{th} \sigma^{\prime \prime}} \frac{d \phi}{d \sigma^{\prime \prime}}-\frac{V^{\prime}(\phi)}{H^{2}}=0 .
$$

Начальные условия для (38) в двух случаях таковы: 




Рис. 8

1) $\left.\phi\right|_{\sigma^{\prime \prime}=0}$ близко к 0 для $\mu \rightarrow+\infty$;

2) $\left.\phi\right|_{\sigma^{\prime \prime}=0}$ близко к $\phi+$ для $\mu \rightarrow-\infty$;

3) $\left.\frac{d \phi}{d \sigma^{\prime \prime}}\right|_{\sigma^{\prime \prime}=0}=0$ для обоих случаев.

Если в уравнении (38) рассматривать $\phi$ как координату материальной точки, а $\sigma^{\prime \prime}$ как время, то оно описывает движение этой материальной точки в потенциале $V(\phi) / H^{2}$ с зависяшим от времени коэффишиентом трения $3 /$ th $\sigma^{\prime \prime}$. Поскольку в любой момент времени коэффициент трения больше трех, точка, имеюшая вначале нулевую скорость, обязательно скатится в ближайший минимум потенциала, если только ее начальное положение отлично от $\phi_{0}$. В первом случае это будет $\phi=0$, во втором $-\phi=\phi_{+}$(рис.9).

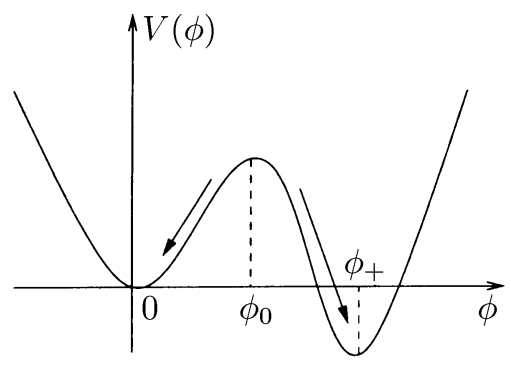

Рис. 9

Таким образом, $\phi \rightarrow 0$ при $\mu \rightarrow+\infty$ и $\phi \rightarrow \phi_{+}$при $\mu \rightarrow-\infty$. Отсюда получаем

$$
\left.\phi\right|_{\eta \rightarrow 0^{-}, \rho>1}=0,\left.\quad \phi\right|_{\eta \rightarrow 0^{-}, \rho<1}=\phi_{+} .
$$

Из последних соотношений следует, что рассматриваемое решение описывает пузырь истинного вакуума в море ложного, асимптотический координатный радиус $\rho$ которого 
равен единище. Пузыри других радиусов могут быть получены из этого "эталонного" пузыря растягиванием координат (заменой $\eta \rightarrow \alpha \eta, \rho \rightarrow \alpha \rho)$, причем главная квазиклассическая экспонента для вероятности их образования не зависит от размера, поскольку действие при таких преобразованиях не меняется.

Вернемся к граничному условию 2. Фигурируюшее в его формулировке поведение координат $s$ и $\psi$ задает согласно (37) следующий закон изменения переменной $\mu$ :

$$
\mu \sim i e^{s} \cos e^{-2 s} \sim i e^{s}, \quad s \rightarrow \infty .
$$

Осталось только аналитически продолжить $\phi$ в область больших мнимых $\mu$ правильным образом. Для этого вернемся к самому началу, а именно вспомним, что продолжать из области действительных $\eta$ в область чисто мнимых $\eta$ (и, следовательно, действительных $\zeta$ ) следует так, как показано на рис. 3 . В отношении $s$ это соответствует изменению от $s_{1}-i(\pi / 2)$ до $s_{1}$, где $s_{1}$ действительно и велико (ср. с рассуждениями, использованными при выводе формулы (27)). При этом $\mu$ описывает бесконечно удаленную дугу, лежашую в первом квадранте (см. рис. 10). При таком продолжении $\lim _{\mu \rightarrow+i \infty} \phi=\lim _{\mu \rightarrow+\infty} \phi=0$ и выполнение условия 2 доказано.

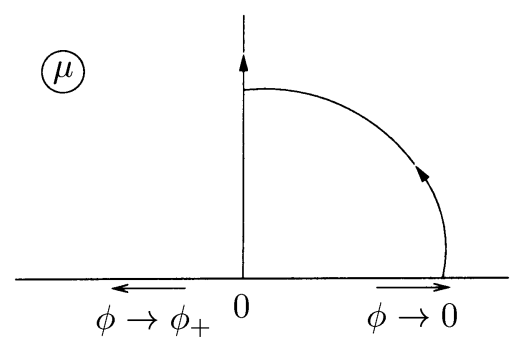

Рис. 10

Таким образом, инстантон Коулмена-де Люччиа действительно описывает распад ложного вакуума в пространстве де Ситтера, причем тогда, когда квантовые флуктуации скалярного поля находятся в состоянии конформного вакуума.

\section{6. ИНСТАНТОН ХОКИНГА-МОССА КАК ПРЕДЕЛ ИНСТАНТОНОВ СО СВЯЗЬЮ}

Рассмотрим теперь ситуацию, когда нетривиального решения уравнений поля на сфере не сушествует. Для того чтобы найти ширину распада ложного вакуума в этом случае, наши предыдушие рассуждения следует модифишировать. Основная идея состоит в том, чтобы вместо настоящего решения уравнений поля найти однопараметрическое семейство почти седловых конфигураций $\phi_{\varepsilon}(\mathbf{x}, \eta)$, удовлетворяющих граничным условиям раздела 3 и дающих вклад в вероятность распада ложного вакуума. Действие на таких конфигурациях зависит от параметра $\varepsilon$, а их вклад в вероятность распада стремится к максимальному при $\varepsilon \rightarrow 0^{+}$. Предельная конфигурация $\phi_{\varepsilon=0}$ (инстантон Хокинга-Мосса) граничным условиям не удовлетворяет (хотя и представляет собой решение уравнений поля), однако действие на ней определяет квазиклассическую экспоненту 
распада. Ситуация в этом случае аналогична той, которая возникает при исследовании инстантонных эффектов в четырехмерных теориях Янга-Миллса-Хиггса [16].

Введем в исходный функциональный интеграл (7) единицу:

$$
\int_{-\infty}^{\infty} d \varepsilon \delta(\varepsilon-\Delta)=\int_{-\infty}^{\infty} d \varepsilon \int_{-\infty}^{\infty} \frac{d \alpha}{2 \pi} e^{i \alpha(\varepsilon-\Delta)}
$$

где

$$
\Delta=\int \frac{d^{3} x d \eta}{2 H^{2} \eta^{2}}\left(\left(\frac{\partial \phi_{1}}{\partial \eta}\right)^{2}+\left(\frac{\partial \phi_{2}}{\partial \eta}\right)^{2}-\left(\frac{\partial \phi_{1}}{\partial x^{i}}\right)^{2}-\left(\frac{\partial \phi_{2}}{\partial x^{i}}\right)^{2}\right)
$$

и изменим порядок интегрирования, сделав интегрирование по $d \varepsilon d \alpha$ внешним. Повторяя с небольшими изменениями цепочку рассуждений разделов 3 и 5 , получаем для ширины распада

$$
\Gamma=\int d \varepsilon \exp \left(-S_{\zeta}\left[\phi_{\varepsilon}\right]\right)
$$

где $S_{\zeta}$ по-прежнему определяется формулой (33), а классические решения $\phi_{\varepsilon}$, кроме всех условий раздела 5, удовлетворяют еще и дополнительному ограничению

$$
\frac{2 \pi^{2}}{H^{2}} \int_{0}^{\pi} d \sigma^{\prime} \sin ^{3} \sigma^{\prime} \frac{1}{2}\left(\frac{\partial \phi_{\varepsilon}}{\partial \sigma^{\prime}}\right)^{2}=\varepsilon
$$

Для вывода уравнения для $\phi_{\varepsilon}$ вычтем из действия (33) связь (42) с множителем Лагранжа $\lambda$ :

$$
\begin{aligned}
S_{\zeta} & -\lambda \frac{2 \pi^{2}}{H^{2}} \int_{0}^{\pi} d \sigma^{\prime} \sin ^{3} \sigma^{\prime} \frac{1}{2}\left(\frac{\partial \phi_{\varepsilon}}{\partial \sigma^{\prime}}\right)^{2}= \\
& =\frac{2 \pi^{2}}{H^{2}}(1-\lambda) \int_{0}^{\pi} d \sigma^{\prime} \sin ^{3} \sigma^{\prime}\left(\frac{1}{2}\left(\frac{\partial \phi_{\varepsilon}}{\partial \sigma^{\prime}}\right)^{2}+\frac{V\left(\phi_{\varepsilon}\right)}{H^{2}(1-\lambda)}\right)
\end{aligned}
$$

и проварьируем по $\phi_{\varepsilon}$. В результате получим уравнение

$$
\frac{d^{2} \phi_{\varepsilon}}{d \sigma^{\prime 2}}+\frac{3}{\operatorname{tg} \sigma^{\prime}} \frac{d \phi_{\varepsilon}}{d \sigma^{\prime}}-\frac{V^{\prime}\left(\phi_{\varepsilon}\right)}{H^{2}(1-\lambda)}=0
$$

Мы видим, что наложение условий связи (42) с точки зрения уравнений поля сводится к эффективному изменению потенциала.

Граничная задача, состоящая из уравнения (44), связи (42) и условий “а", "б” и “в" раздела 5 , имеет решение при любых достаточно малых и положительных $\varepsilon$. Чтобы в этом убедиться, заметим, что решение, удовлетворяюшее (44) и условиям “а”, "б”, “в", существует, если

$$
\left|\frac{V^{\prime \prime}\left(\phi_{0}\right)}{H^{2}(1-\lambda)}\right|>4
$$

(cp. с (36)). При

$$
(1-\lambda) \rightarrow\left|\frac{V^{\prime \prime}\left(\phi_{0}\right)}{4 H^{2}}\right|
$$


это решение стремится к константе, интеграл от квадрата его производной стремится к нулю, поэтому надлежащим выбором $\lambda$ всегда можно добиться выполнения условия (42). На основании результатов раздела 5 при фиксированном $\varepsilon>0$ решение $\phi_{\varepsilon}, \mathrm{c}$ одной стороны, описывает после аналитического продолжения в физическое пространство образование пузыря истинного вакуума. С другой стороны, при $\varepsilon \rightarrow 0^{+}$конфигурация $\phi_{\varepsilon}$ тождественно обращается в постоянное поле $\phi=\phi_{0}$, т.е. переходит в инстантон Хокинга-Мосса. Известно $[7,12]$, что в предполагаемой в настоящем разделе ситуации последний дает безусловный минимум действия $S_{\zeta}$, по крайней мере, в классе $O(4)$-симметричных полевых конфигураций. Таким образом, $S_{\zeta}\left[\phi_{\varepsilon}\right]$ как функция $\varepsilon$ имеет минимум при $\varepsilon=0$, и, следовательно, наибольший вклад в ширину (41) вносят решения $\phi_{\varepsilon}$, соответствуюшие мальм $\varepsilon$. Взяв в качестве значения интеграла (41) величину подынтегрального выражения в седловой точке, получим

$$
\Gamma \propto e^{-S_{\zeta}\left[\phi_{0}\right]}
$$

где в показателе экспоненты стоит действие инстантона Хокинга-Мосса

$$
S_{\zeta}\left[\phi_{0}\right]=\frac{8 \pi^{2}}{3 H^{4}} V\left(\phi_{0}\right) .
$$

Итак, инстантон Хокинга-Мосса представляет собой предельньй случай инстантонов со связью $\phi_{\varepsilon}(\mathbf{x}, \eta)$. При $\varepsilon>0$ конфигурация $\phi_{\varepsilon}(\mathbf{x}, \eta)$ имеет ту же структуру, что и инстантон Коулмена-де Люччиа, рассмотренный в предыдушем разделе. Таким образом, распад ложного вакуума вновь происходит путем образования в нем пузырей новой фазы.

\section{7. ПРЫЖКИ ВВЕРХ}

Остановимся еще на одной любопытной особенности теории скалярного поля в пространстве де Ситтера, качественно отличающей ее от теории в плоском пространстве. В пространстве де Ситтера возможны самопроизвольные туннельные переходы из истинного вакуума в ложный; возможность прыжков вверх из-за квантовых флуктуаций скалярного поля отмечалась в работе [17] в связи с инфляционными теориями. Необходимая для этого энергия черпается из гравитационного поля, которое при используемых в данной статье предположениях вообше представляет собой бесконечный резервуар энергии. Описание такого рода процессов естественно возникает в рамках предложенного нами подхода.

Действительно, заметим, что при введении координат $\sigma^{\prime}, \psi^{\prime}$ (см. раздел 5) мы могли бы вместо замены (32) использовать другую замену:

$$
\cos \sigma^{\prime}=\sin \sigma \cos \left(\psi-\psi_{0}\right), \quad \sin \sigma^{\prime} \cos \psi^{\prime}=\cos \sigma
$$

где $\psi_{0}$ - параметр, изменяющийся от 0 до $\pi$. Это соответствовало бы переносу начала системы отсчета на сфере в точку $\sigma=\pi / 2, \psi=\psi_{0}$. В такой системе координат снова существует $O(4)$-симметричное решение уравнения $(30)$, зависяшее только от $\sigma^{\prime}$ и как функция от $\sigma^{\prime}$ полностью совпадаюшее с решением, рассмотренным в разделе 5 . (Мы для определенности рассматриваем ситуацию, когда нетривиальное решение существует, перенесение изложенного ниже на случай Хокинга-Мосса очевидно.) Однако 
при произвольном выборе $\psi_{0}$ это решение не имеет физического смысла. Это видно из того, что его аналитическое продолжение в область действительных $\rho$ и $\eta$ не обладает необходимым для гладкости свойством

$$
\left.\frac{\partial \phi}{\partial \rho}\right|_{\rho=0}=0
$$

Действительно, введя, как и в разделе 5 , переменную $\mu=\cos \sigma^{\prime}$, которая теперь связана с $s^{\prime}$ и $\psi$ соотношением

$$
\mu=\operatorname{ch} s^{\prime} \cos \left(\psi-\psi_{0}\right)
$$

(ср. с (37)), и используя формулы (23), получаем

$$
\left.\frac{\partial \phi}{\partial \rho}\right|_{\rho=0}=\left.\frac{e^{s^{\prime}}}{\operatorname{ch} s^{\prime}} \frac{\partial \phi}{\partial \psi}\right|_{\psi=\pi}=-\left.\operatorname{ch} s^{\prime} \sin \left(\pi-\psi_{0}\right) \frac{\partial \phi}{\partial \mu}\right|_{\mu=\operatorname{ch} s^{\prime} \cos \left(\pi-\psi_{0}\right)},
$$

что не равно нулю, если $\psi_{0} \neq 0, \pi$. Таким образом, указанное решение может описывать некоторый физический процесс, только если $\psi_{0}$ равно 0 или $\pi$. Первая возможность рассмотрена нами в разделе 5. Теперь сконцентрируемся на второй. Вместо (37) в этом случае имеем

$$
\mu=-i \operatorname{sh} s \cos \psi=-\operatorname{ch}^{\prime} \cos \psi
$$

и, следовательно (ср. с (39)),

$$
\lim _{\eta \rightarrow 0^{-}, \rho>1} \phi=\lim _{\mu \rightarrow-\infty} \phi=\phi_{+}, \quad \lim _{\eta \rightarrow 0^{-}, \rho<1} \phi=\lim _{\mu \rightarrow+\infty} \phi=0, \quad \lim _{s \rightarrow \infty, \phi \sim e^{-2 s}} \phi=\phi_{+} .
$$

Точно такие же соотношения мы получили бы, если бы с самого начала рассматривали переходы из истинного вакуума в ложный, а не наоборот. Это дает нам право утверждать, что решение с $\psi_{0}=\pi$ описывает образование пузыря ложного вакуума в истинном. Вероятность этого процесса по-прежнему определяется действием (33), из которого для получения полного показателя квазиклассической экспоненты нужно вычесть действие на тривиальном решении, соответствуюшем истинному вакууму:

$$
\frac{2 \pi^{2}}{H^{4}} \int_{0}^{\pi} d \sigma^{\prime} \sin ^{3} \sigma^{\prime} V\left(\phi_{+}\right)=\frac{8 \pi^{2}}{3 H^{4}} V\left(\phi_{+}\right)
$$

Отсюда находим вероятность образования пузыря:

$$
\Gamma \propto \exp \left[-S_{\zeta}+\frac{8 \pi^{2}}{3 H^{4}} V\left(\phi_{+}\right)\right]
$$

Предельный переход к плоскому пространству осушествляется устремлением постоянной Хаббла к нулю. При этом $S_{\zeta}$ стремится к постоянной величине, определяюшей ширину распада ложного вакуума в плоском пространстве; поскольку же $V\left(\phi_{+}\right)<0$, вероятность скачка из истинного вакуума в ложный, как и следовало ожидать, экспоненциально исчезает. 


\section{8. ЗАКЛЮЧЕНИЕ}

В настоящей статье мы применили метод функционального интегрирования к задаче о распаде ложного вакуума во внешнем пространстве де Ситтера. При этом мы ограничились теорией одного скалярного поля и выбором конформного вакуума скалярных частиц над классическим ложным вакуумом в качестве определения квантового ложного вакуума. При этих условиях мы из первых принципов воспроизвели результаты Коулмена-де Люччиа и Хокинга-Мосса относительно главного квазиклассического приближения для ширины распада.

Инстантон Хокинга-Мосса мы интерпретировали как предел инстантонов со связью и показали, что хотя он и определяет вероятность распада ложного вакуума, вид поля после туннелирования задается не им, а близкими к нему конфигурациями. При этом результатом туннелирования является, как обычно, пузырь истинного вакуума.

Было исследовано еше одно интересное явление - туннельные переходы из истинного вакуума в ложный, и вычислена вероятность такого процесса. Она, как и следовало ожидать, экспоненциально стремится к нулю при предельном переходе к плоскому пространству.

Хотя в этой статье обсуждался лишь вполне определенный класс процессов, предложенный подход, явно учитывающий начальные состояния квантового поля (в нашем случае это был конформный вакуум), очевидным образом обобщается на другие квазиклассические процессы в искривленном пространстве-времени. Ключевые для нашего подхода граничные условия (13) и (14) имеют в действительности весьма общий характер, если под положительной частотностью понимать асимптотические свойства решения $\phi(x)$ по отношению к такому набору полевых мод, который соответствует выбранному вакууму квантового поля. Другое дело, что конкретные аналитические продолжения решения в область комплексных координат, использованные в этой работе, являются, по-видимому, спешифичными для пространства-времени де Ситтера и конформного вакуума скалярных частиц.

В заключение отметим, что в пространстве-времени де Ситтера возможен выбор системы координат, отличающейся от (2), и соответственно выбор вакуума скалярных частищ, отличаюшегося от конформного вакуума. Так, в работе [18] распад ложного вакуума рассмотрен в приближении внешней метрики в статической системе координат и воспроизведены результаты Коулмена-де Люччиа и Хокинга-Мосса. В то же время вакуум, определенный в статической системе координат, не инвариантен даже относительно трансляций [9] и, следовательно, отличен от вакуума, рассмотренного в нашей работе. Поэтому совпадение результатов настоящей работы и работы [18] представляется удивительным. Надо, однако, отметить, что в [18] понятие вакуума вообще явно не сформулировано. Поэтому мы предполагаем, что указанное совпадение объясняется следующим образом: в работе [18] на самом деле эффективно рассматривается туннелирование не из вакуума статической системы координат, а из сильно возбужденного состояния, которым является в статической системе координат конформный вакуум. Данный вопрос нуждается в более детальном изучении.

Мы надеемся, что настоящая работа проливает свет на некоторые особенности метода функционального интегрирования в искривленном пространстве-времени и открывает пути для применения этого метода при исследовании различных квазиклассических 
процессов.

Благодарности. Авторы благодарны В.А. Кузьмину, П. Г. Тинякову и С.В. Троицкому за полезные обсуждения. Работа выполнена при частичной поддержке Российского фонда фундаментальных исследований, грант № 99-02-18410.

\section{Список литературы}

[1] М. Б. Волошин, И. Ю. Кобзарев, Л. В. Окунь. ЯФ. 1974. Т. 20. С. 1229.

[2] S. Coleman. Phys. Rev. D. 1977. V. 15. P. 2929.

[3] C. G. Callan, S. Coleman. Phys. Rev. D. 1977. V. 16. P. 1792.

[4] S. Coleman, V. Glaser, A. Martin. Commun. Math. Phys. 1978. V. 58. P. 211.

[5] S. Coleman, F. De Luccia. Phys. Rev. D. 1980. V. 21. P. 3305.

[6] A.H. Guth, E. Weinberg. Nucl. Phys. B. 1983. V. 212. P. 321.

[7] S. W. Hawking, I. G. Moss. Nucl. Phys. B. 1983. V. 224. P. 180.

[8] А. А. Гриб, С.Г. Мамаев, В. М. Мостепаненко. Квантовые эффекты в интенсивных внешних полях. М.: Атомиздат, 1980.

[9] Н. Биррелл, П. Девис. Квантовые поля в искривленном пространстве-времени. М.: Мир, 1984.

[10] A. D. Linde. Nucl. Phys. B. 1983. V. 216. P. 421.

[11] A. A. Старобинский. Космологические модели с промежуточной де ситтеровской стадией: теория и наблюдательные следствия. В сб.: Фундаментальные взаимодействия. Отв. ред. В. Н. Пономарев. М.: МГПИ, 1984. С. 55.

[12] А.С. Гончаров, А.Д. Линде. ЭЧАЯ. 1986. Т. 17. С. 837.

[13] С. Хокинг, Джс. Эллис. Крупномасштабная структура пространства-времени. М.: Мир, 1977.

[14] A. Д. Линде. Физика элементарных частиц и инфляционная космология. М.: Наука, 1990.

[15] V. A. Rubakov, D. T. Son, P. G. Tinyakov. Phys. Lett. B. 1992. V. 278. P. 279; A. N. Kuznetsov, P. G. Tinyakov. Phys. Rev. D. 1997. V. 56. P. 1156.

[16] I. Affleck. Nucl. Phys. B. 1981. V. 191. P. 429.

[17] A. D. Linde. Phys. Lett. B. 1983. V. 131. P. 330.

[18] N. Deruelle. Mod. Phys. Lett. A. 1989. V. 4. P. 1297. 